\title{
Characterization of helical Luttinger liquids in microwave stepped-impedance edge resonators
}

\author{
Alexandre Gourmelon, Hiroshi Kamata $\odot$, Jean-Marc Berroir $\odot$, Gwendal Fève $\odot$, Bernard Plaçais $\odot$, and Erwann Bocquillon $\odot^{*}$ \\ Laboratoire de Physique de l'École Normale Supérieure, ENS, PSL Research University, CNRS, Sorbonne Université, Université Paris \\ Diderot, Sorbonne Paris Cité, 24 rue Lhomond, 75231 Paris Cedex 05, France
}

(Received 21 August 2020; accepted 11 November 2020; published 17 December 2020)

\begin{abstract}
Coulomb interaction has important consequences for the physics of quantum spin Hall edge states, weakening the topological protection via two-particle scattering and renormalizing both the velocity and the charge of collective plasmon modes compared to that of free electrons. Despite these effects, interactions remain difficult to quantify. We propose here simple and robust edge resonator geometries to characterize Coulomb interaction by means of high-frequency measurements. They rely on a transmission line approach and take advantage of the impedance mismatch between the edge states and their microwave environment.
\end{abstract}

DOI: 10.1103/PhysRevResearch.2.043383

The helical edge states of two-dimensional (2D) topological insulators, which exhibit the quantum spin Hall (QSH) effect [1], offer an exciting playground for exotic topological physics such as spin-polarized edge transport [2-4] or topological superconductivity $[5,6]$. Another point of interest is the study of Coulomb interactions, which are particularly prominent in one-dimensional systems. In 2D topological insulators, one-particle scattering is, in principle, suppressed by time-reversal symmetry. However two-particle interactions are constrained, but not forbidden [7], and could have important consequences. They could, for example, generate back-scattering via diverse mechanisms [8-10]. In particular, inter-channel interaction leads to a modification of the charge but also to the spin polarization of the edge plasmons, thus potentially degrading the performance of spin(orbi)tronics devices $[11,12]$ and obscuring the observation of topological transport.

At a microscopic level, Coulomb interaction strongly alters the dynamics of QSH helical edge states. First, the electron Fermi velocity $v_{F}$ is renormalized to a larger value $v$ under the action of Coulomb repulsion. Second, as charges propagate in one edge channel, interchannel interaction drags a small amount of charge in the channel of opposite direction, leading to a nontrivial effective charge of the plasmon modes, reduced from that of the electron by a factor of $\sqrt{K} \in[0,1]$, where $K$ is called the Luttinger parameter. The two parameters $K$ and $v$ fully characterize the dynamical properties of helical edge channels, but remain experimentally hardly accessible. In particular, $K$ is quite elusive: the dc conductance of an ohmically contacted $1 D$ system does not depend on $K[13,14]$, which is thus primarily accessed via power law exponents (tunneling density of states [15] and temperature dependence

*erwann.bocquillon@ens.fr

Published by the American Physical Society under the terms of the Creative Commons Attribution 4.0 International license. Further distribution of this work must maintain attribution to the author(s) and the published article's title, journal citation, and DOI. of the conductance $[16,17])$ or current correlations $[18,19]$. The ac conductance also inherits a $K$ dependence, but studies have concentrated mostly on the low-frequency regime [20], which does not capture the velocity $v$, on more complex setups [21], or on chiral edge modes [22,23].

High-frequency experiments have proven very adequate in the context of the quantum Hall effect to investigate chiral edge magnetoplasmons [24-35]. In the same spirit, we establish in this paper that the scattering of microwaves on capacitively coupled resonators offers a straightforward characterization of both $v$ and $K$. The high-impedance edge channels are then confined between low-impedance input and output circuitry. The impedance mismatch generates reflection at each interface, in a geometry analogous to so-called stepped-impedance resonators, heavily used in acoustics or microwave design. This geometry is advantageous for several reasons: (i) the use of high-frequency $(>10 \mathrm{GHz}$ ) transport allows for using short devices $(\sim 10 \mu \mathrm{m})$ in the ballistic limit; (ii) the capacitive coupling circumvents complications due to ohmic contacts and contact resistances which bring dissipation [36] and have a complex behavior in the GHz range [37]; and (iii) the geometry can be analyzed in terms of microwave networks [38], combining simple experimental setups [39-41] and straightforward interpretation.

\section{EDGE CHANNELS AND EQUIVALENT TRANSMISSION LINES}

In this section we introduce the bosonized Luttinger representation of the helical edge states and establish its relation to a microwave transmission, following previous works [42,43]. For helical edge channels, the bosonized Hamiltonian is formally identical to that of a spinless Luttinger liquid and reads as follows $[7,44]$ :

$$
H=\frac{v}{2 \pi} \int d x\left[\frac{1}{K}\left(\partial_{x} \phi\right)^{2}+K\left(\partial_{x} \theta\right)^{2}\right]-e U \partial_{x} \phi,
$$

where the fields $\phi$ and $\theta$ are related to the right $\left(\phi_{R}\right)$ and left $\left(\phi_{L}\right)$ bosonic fields representing right- and left-movers, with $\phi=\phi_{R}+\phi_{L}$ and $\theta=\phi_{R}-\phi_{L} . U$ is an external potential, 

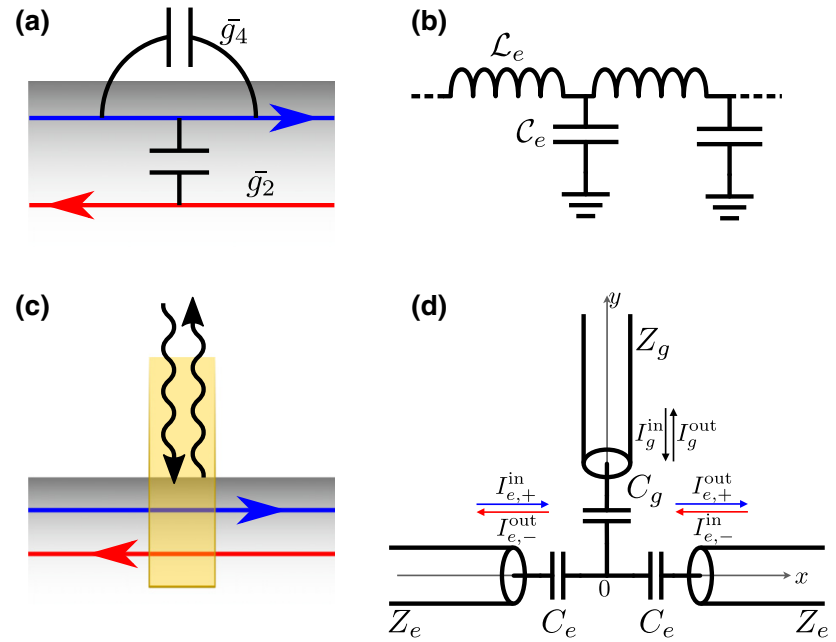

FIG. 1. Schematics of the setup. (a) Schematic view of inter- and intrachannel Coulomb interaction in helical edge states (in red and blue solid lines), with their respective coupling constants $\overline{g_{2}}$ and $\overline{g_{4}}$. (b) Equivalent transmission line representation, with line inductance $\mathcal{L}_{e}$ and line capacitance $\mathcal{C}_{e}$. (c) Schematic view of a gate coupling capacitively to helical edge states. (d) Equivalent transmission line representation, with geometric capacitance $C_{g}$ and quantum capacitance $C_{e}$.

in a minimal coupling approach. Coulomb interaction renormalizes the sound velocity of the collective excitation $v$ and defines the Luttinger parameter $K$, which are expressed as a function of the Fermi velocity $v_{F}$ and the interchannel (respectively, intrachannel) coupling constants $\overline{g_{2}}$ (respectively, $\left.\overline{g_{4}}\right)$ :

$$
\begin{gathered}
v=v_{F} \sqrt{\left(1+\overline{g_{4}}+\overline{g_{2}}\right)\left(1+\overline{g_{4}}-\overline{g_{2}}\right)}, \\
K=\frac{\sqrt{1+\overline{g_{4}}-\overline{g_{2}}}}{\sqrt{1+\overline{g_{4}}+\overline{g_{2}}}} .
\end{gathered}
$$

In the absence of external potential $(U=0)$, this Hamiltonian is completely equivalent to that of an $L C$ distributed transmission line [38] with line capacitance $\mathcal{C}_{e}$ and line inductance $\mathcal{L}_{e}$, such as the one sketched in Fig. 1(b):

$$
\begin{aligned}
\mathcal{H} & =\frac{\rho_{e}^{2}}{2 \mathcal{C}_{e}}+\frac{1}{2} \mathcal{L}_{e} I_{e}^{2}, \\
\text { with } \mathcal{C}_{e} & =\frac{2 K}{R_{K} v}, \quad \mathcal{L}_{e}=\frac{R_{K}}{2 K v},
\end{aligned}
$$

where $\rho_{e}=\frac{e}{\pi} \partial_{x} \phi$ is the charge density, $I_{e}=\frac{e v K}{\pi} \partial_{x} \theta$ is the current flowing on the edge, and $R_{K}$ is the quantum of resistance. This identification establishes the equivalence between a helical Luttinger liquid and a transmission line with characteristic impedance $Z_{e}$ and velocity $v$ given by [45]

$$
Z_{e}=\sqrt{\frac{\mathcal{L}_{e}}{\mathcal{C}_{e}}}=\frac{R_{K}}{2 K}, \quad v=\frac{1}{\sqrt{\mathcal{L}_{e} \mathcal{C}_{e}}} .
$$

This transmission line hosts two modes with linear dispersion and velocity $v$, propagating in opposite directions (denoted $\pm)$. Here, one can interpret the factor $K$ as a reduced effective charge $\sqrt{K} e$ affecting $\mathcal{L}_{e}$ and $\mathcal{C}_{e}$ and, in turn, the line impedance $Z_{e}$.
One can introduce the currents $I_{e}(x, t)$ and the voltages $V_{e}(x, t)=\rho_{e}(x, t) / \mathcal{C}_{e}[38,43]:$

$$
\begin{aligned}
& V_{e}(x, t)=\int \frac{d \omega}{2 \pi}\left(V_{e}^{-} e^{j \omega\left(t+\frac{\omega}{v} x\right)}+V_{e}^{+} e^{j \omega\left(t-\frac{\omega}{v} x\right)}\right), \\
& I_{e}(x, t)=\int \frac{d \omega}{2 \pi}\left(-I_{e}^{-} e^{j \omega\left(t+\frac{\omega}{v} x\right)}+I_{e}^{+} e^{j \omega\left(t-\frac{\omega}{v} x\right)}\right),
\end{aligned}
$$

such that $Z_{e}=\frac{V_{e}^{ \pm}}{I_{e}^{ \pm}}$.

In this framework, the currents $I_{e}^{ \pm}$carried by both modes \pm then simply acquire a phase factor of $s_{ \pm}=e^{ \pm i \frac{\omega}{v}\left(x-x^{\prime}\right)}$ as they propagate from point $x$ to $x^{\prime}$.

\section{CAPACITIVE COUPLING IN A TRANSMISSION LINE APPROACH}

This transmission line approach allows for a simple description of elaborate geometries, in close analogy to microwave network analysis. Here we start by describing capacitive contacts. The latter are essentially dissipationless, thus circumventing the dissipation inherent to the Landauer contact resistance. Besides, such reactive contacts achieve a simple capacitive coupling even at high frequencies, unlike ohmic contacts which exhibit mixed capacitive and resistive behaviors [37]. They have been heavily used in quantum Hall high-frequency experiments $[22,24,31,46]$ and have triggered recent interest in Hall gyrators and circulators [34-36,47]. The capacitive contact is described as a three-port device coupling at $(x, y)=(0,0)$ the edge states (seen as two semiinfinite transmission lines of impedance $Z_{e}$, for $x>0$ and $x<0$ ) to the gate (seen as a semi-infinite transmission line of impedance $Z_{g}=50 \Omega$ along the $y>0$ axis), in the geometry shown in Fig. 1(d). The geometrical gate capacitance is denoted as $C_{g}$, while the quantum capacitance effects are accounted for by $C_{e}$. This pointlike description is inspired by transmission line models, but similar results have been obtained with a more elaborate plasmon distributed model, adding some finite-size effects which are disregarded here (see more in Sec. V, Experimental considerations).

Introducing the Fourier modes $I_{g}^{ \pm}$and $V_{g}^{ \pm}$for the gate transmission line, one obtains from Kirchhoff's laws at the coupling point $(x, y)=(0,0)$ the relations between input and output currents in each of the arms:

$$
\begin{gathered}
I_{g}^{\mathrm{in}}-I_{g}^{\mathrm{out}}=\sum_{i= \pm}\left(I_{e, i}^{\mathrm{out}}-I_{e, i}^{\mathrm{in}}\right), \\
\forall i= \pm, \Lambda_{e} I_{e, i}^{\mathrm{out}}-\Lambda_{e}^{*} I_{e, i}^{\mathrm{in}}=\frac{C_{e}}{C_{g}}\left(\Lambda_{g} I_{g}^{\mathrm{out}}-\Lambda_{g}^{*} I_{g}^{\mathrm{in}}\right),
\end{gathered}
$$

where $I_{g}^{\text {in/out }}=\left.I_{g}^{ \pm}\right|_{y=0^{+}}, I_{e, \pm}^{\text {in }}=\left.I_{e}^{ \pm}\right|_{x=0^{\mp}}$, and $I_{e, \pm}^{\text {out }}=\left.I_{e}^{ \pm}\right|_{x=0^{ \pm}}$ [see Fig. 1(d)], and $\Lambda_{e}(\omega)=1+j \omega Z_{e} C_{e}$ and $\Lambda_{g}(\omega)=1+$ $j \omega Z_{g} C_{g}$. Solving these three equations yields the scattering matrix elements, relating outgoing to ingoing currents [48]:

$$
\begin{gathered}
S_{g g}=\frac{1+\frac{2 C_{e}}{C_{g}} \frac{\Lambda_{g}^{*}(\omega)}{\Lambda_{e}(\omega)}}{1+\frac{2 C_{e}}{C_{g}} \frac{\Lambda_{g}(\omega)}{\Lambda_{e}(\omega)}}, \\
S_{g \pm}=\frac{2 j \omega Z_{e} C_{e} \frac{1}{\Lambda_{e}(\omega)}}{1+\frac{2 C_{e}}{C_{g}} \frac{\Lambda_{g}(\omega)}{\Lambda_{e}(\omega)}}=\frac{Z_{e}}{Z_{g}} S_{ \pm g},
\end{gathered}
$$




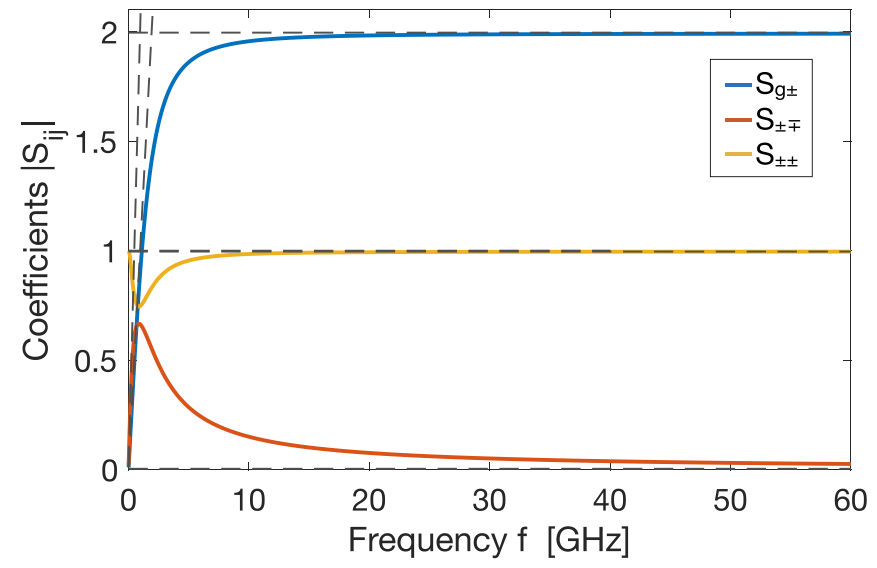

FIG. 2. Scattering coefficients of a capacitive contact: $\left|S_{g \pm}\right|$ is represented as a blue line, $\left|S_{\mp \pm}\right|$ as a red line, and $\left|S_{ \pm \pm}\right|$as a yellow line. For each coefficient, the gray dashed lines indicate the low- and high-frequency asymptotes obtained analytically. The graphs were obtained with the following parameters: $C_{e}=16 \mathrm{fF}, C_{g}=8 \mathrm{fF}$, and $K=0.5$.

$$
\begin{gathered}
S_{ \pm \pm}=\frac{\Lambda_{e}^{*}(\omega)}{\Lambda_{e}(\omega)} \frac{1+\frac{2 \Lambda_{g}(\omega)}{\Lambda_{e}^{*}(\omega)} \frac{C_{e}}{C_{g}}}{1+\frac{2 \Lambda_{g}(\omega)}{\Lambda_{q}(\omega)} \frac{C_{e}}{C_{X}}}, \\
S_{ \pm \mp}=\frac{C_{e}}{C_{g}} \frac{\Lambda_{g}(\omega)}{\Lambda_{e}^{2}(\omega)} \frac{2 j \omega Z_{e} C_{e}}{1+\frac{2 C_{e}}{C_{g}} \frac{\Lambda_{g}(\omega)}{\Lambda_{e}(\omega)}} .
\end{gathered}
$$

The moduli of $S_{g \pm}, S_{ \pm \pm}$, and $S_{ \pm \mp}$ are plotted in Fig. 2 as a function of the frequency $f=\frac{\omega}{2 \pi}$, for a realistic set of parameters (see Sec. V, Experimental considerations). In these coefficients, two $R C$ timescales appear, namely, the gate one, $Z_{g} C_{g}$, and that of the edge states, $Z_{e} C_{e}$, defining two regimes: low frequencies for which $\omega \ll 1 / Z_{g} C_{g}$ and $1 / Z_{e} C_{e}$ and high frequencies for which $\omega \gg 1 / Z_{g} C_{g}$ and $1 / Z_{e} C_{e}$. In the low-frequency regime, the capacitive elements $C_{g}$ and $C_{e}$ play the dominant role. We in particular observe that $S_{g \pm} \simeq$ $2 j \omega Z_{e} C_{e}$ and $S_{ \pm \mp} \simeq j \omega \frac{C_{e}}{C_{g}} C_{t}$, with $C_{t}=\frac{2 C_{e} C_{g}}{C_{g}+2 C_{e}}$ being the total capacitance.

In contrast, at high frequency, the capacitors are transparent and do not play any role. The scattering elements are thus dominated by the impedance mismatch [38] between the edge states (impedance $Z_{e}$ ) and the gate (impedance $Z_{g}$ ) with, for instance, $S_{g \pm}=\frac{2 Z_{e}}{Z_{e}+2 Z_{g}} \simeq 2$. This high-frequency limit of transport and the role of the low-impedance environment are both often disregarded but play here a crucial role. Indeed, as we see below, their interplay through impedance mismatch provides a very straightforward approach to measuring $Z_{e}$ and thus $K$.

\section{LINEAR AND RING RESONATORS}

In this section, we introduce the geometry of linear and ring "resonators" in which the two edge states are confined between two capacitive contacts (Fig. 3). In the linear resonator [Fig. 3(a)], the contacts are separated by a distance $d$ and we assume that the regions outside the contacts are semi-infinite and sink all incoming waves. In the ring geom- (a)

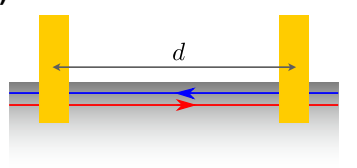

(c)

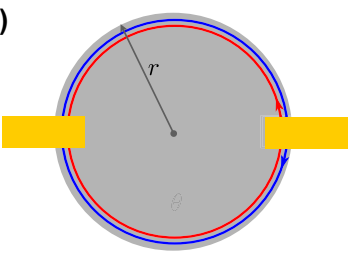

(b)

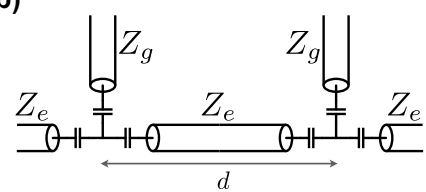

(d)

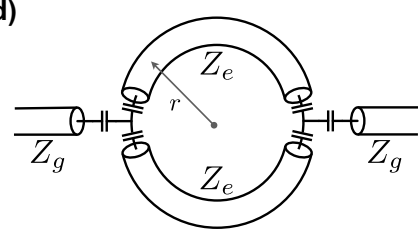

FIG. 3. Geometry of the linear and ring resonators. (a),(c) Schematic view of a linear resonator (a) and a ring resonator (c) for the edge states of the QSH effect (in red and blue solid lines). Capacitive contacts are depicted in yellow. (b),(d) Equivalent transmission line representation of the linear (b) and ring (d) resonators.

etry [Fig. 3(c)], the edge states circulate along a disk-shaped mesa of radius $r$, with the two contacts placed symmetrically on either side of the disk. Each structure forms a two-port device, with equivalent transmission line representations depicted in Figs. 3(b) and 3(d). The schemes in Figs. 3(b) and 3(d) highlight the analogy with stepped-impedance resonators, with regions of the impedance $Z_{e}$ located between ports of the lower impedance $Z_{g}$. Using the scattering matrix of the free QSH edge and of the capacitive contact, we compute the transmission $T$ of each device and analyze it below. The reflexion coefficient on each contact is disregarded as it is mostly dominated by $S_{g g} \simeq 1$, and we assume for now that the devices are ballistic.

Starting with the linear resonator, summing the contributions of all waves, one obtains the transmission:

$$
T=\frac{S_{g+} S_{+g} e^{-j \omega \frac{d}{v}}}{1-S_{+-} S_{-+} e^{-2 j \omega \frac{d}{v}}}=\frac{Z_{g}}{Z_{e}} \frac{S_{g+}^{2} e^{-j \omega \frac{d}{v}}}{1-S_{+-}^{2} e^{-2 j \omega \frac{d}{v}}} .
$$

$T$ exhibits the familiar form of a Fabry-Pérot (FP) resonator: the numerator reflects the direct path from one gate to the other, while the denominator encodes the multiple roundtrips in the cavity. The modulus $|T|$ and the phase $\arg (T)$ are plotted in Figs. 4(a), 4(b) for $K=0.25,0.5$, and 1. We first note that, since $S_{+-}$remains small, the FP oscillations are quite weak, and most of the excitation signal leaks to the regions located beyond both capacitive contacts. The transmission $T$ is thus governed by its numerator [plotted as a gray dashed line in Fig. 4(a)]. At low frequencies (for $\omega \ll 1 / Z_{g} C_{g}$ and $\left.1 / Z_{e} C_{e}\right), T$ is quite low, dominated by the succession of two capacitive elements $S_{g+}^{2}$, with $|T| \propto \omega^{2}$. The physics of interactions remains mostly inaccessible. For the opposite situation, at high-frequencies, $|T|$ saturates at a maximum value $\left|T_{\text {sat }}\right|$ imposed by the saturation of $S_{g+}$, with $\left|T_{\text {sat }}\right|=$ $\frac{4 Z_{g}}{Z_{g}}=\frac{8 K Z_{g}}{R_{K}}$. In this regime, $S_{g+}$ is real, such that $\arg (T) \simeq \frac{\omega d}{v}$. This simple geometry thus allows for a very direct readout of the velocity $v$ from $\arg (T)$, and of the Luttinger interaction parameter $K$ via $\left|T_{\text {sat }}\right|$. 
(a)

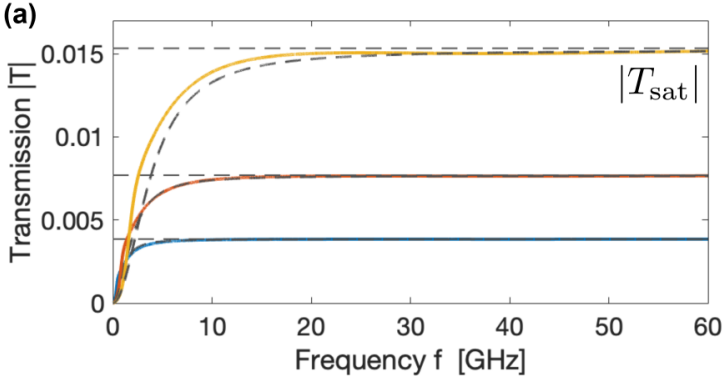

(b)

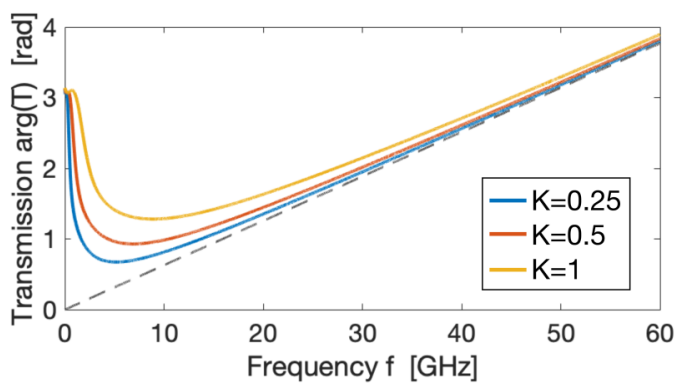

(c)

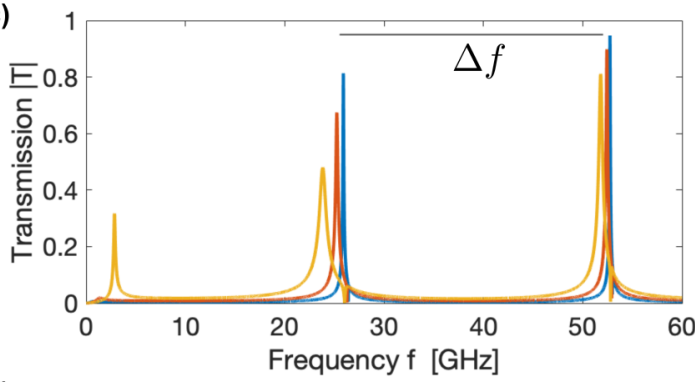

(d)

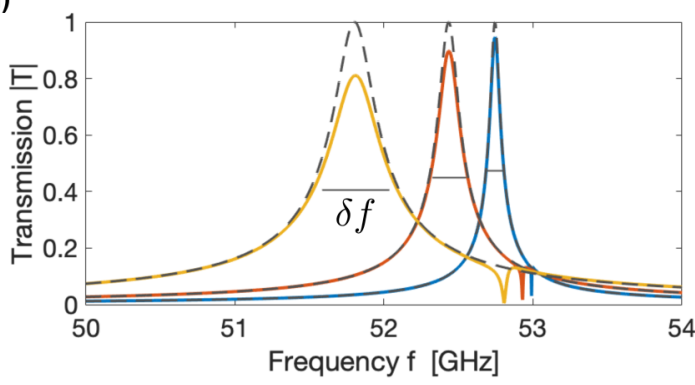

FIG. 4. Transmission for the linear and ring resonators. (a),(b) For $K=0.25,0.5$, and 1, the moduli $|T|$ [panel (a)] and the phase $\arg (T)$ [panel (b)] of the transmission of the linear resonators are plotted as solid colored lines. In panel (a), the dashed lines represent the approximation $\frac{Z_{g}}{Z_{e}}\left|S_{g+}\right|^{2}$ and the high-frequency asymptote $\left|T_{\text {sat }}\right|$, allowing one to determine $K$. In panel (b), the dashed line represents the asymptote $\omega d / v$, giving access to $v$. (c),(d) For $K=0.25,0.5$, and 1, the moduli $|T|$ of the transmission of the ring resonators are plotted as solid colored lines. In panel (c), the solid gray line represents the interval $\Delta f$ between two resonances. In panel (d), the dashed lines represent the approximation given in Eq. (17), while the solid gray lines represent the peak widths $\delta f$, allowing one to determine $K$. All graphs were obtained with the following parameters: $C_{e}=16 \mathrm{fF}, C_{g}=8 \mathrm{fF}, v=1 \times 10^{6} \mathrm{~ms}^{-1}, d=10 \mu \mathrm{m}$, and $r=6 \mu \mathrm{m}$.

We now move on to the case of the ring resonator. In this case, many waves, scattering on either of the contacts, interfere and contribute to the output current. The transmission $T$ can be analytically derived in full generality and reads as follows:

$$
T=\frac{2 Z_{g}}{Z_{e}} \frac{S_{g+}^{2} e^{-j \omega \frac{\pi r}{v}}\left(1-e^{-2 j \omega \frac{d}{v}}\left(S_{++}^{2}-S_{+-}^{2}\right)\right)}{\left(1-2 e^{-j \omega \frac{d}{v}} S_{+-}-e^{-j \omega \frac{2 \pi r}{v}}\left(S_{++}^{2}-S_{+-}^{2}\right)\right)\left(1+2 e^{-j \omega \frac{\pi r}{v}} S_{+-}-e^{-j \omega \frac{2 \pi r}{v}}\left(S_{++}^{2}-S_{+-}^{2}\right)\right)} .
$$

As can be seen from Figs. 4(c) and 4(d), $T$ exhibits many features, with a quite complex behavior. Nonetheless, in the high-frequency regime $\omega \gg 1 / Z_{g} C_{g}$ and $1 / Z_{e} C_{e}$, the different $S_{i j}$ coefficients are all real, and $S_{+-}$is rather small, so that $T$ can be approximated by the following formula (plotted as a dashed gray line):

$$
T=\frac{2 Z_{g}}{Z_{e}} \frac{S_{g+}^{2} e^{-j \omega \frac{\pi r}{v}}}{1-e^{-j \omega \frac{2 \pi r}{v}} S_{++}\left(S_{++}+2 S_{+-}\right)} .
$$

In this regime, one again recognizes a familiar Fabry-Pérot resonator, with an effective "mirror" reflection coefficient $\Gamma=$ $S_{++}\left(S_{++}+2 S_{+-}\right)$. Equidistant resonance peaks are separated by $\Delta f=\frac{v}{2 \pi r}$, the round-trip frequency, allowing for a simple extraction of $v$. Here, losses are ignored, and the geometry is closed. As a consequence, the amplitude of the peaks tends to 1 in the high-frequency regime, regardless of parameters. However, the impedance $Z_{e}$ acts on the value of $S_{ \pm \pm}$and hence on the reflexion $\Gamma$ which determines the peak width: we find that the width of the peaks (full width at half maximum) is given by $\delta f=\frac{v}{2 \pi r} \frac{16 \sqrt{3} K Z_{g}}{\pi R_{K}}$, allowing for determining $K$. One can also simultaneously evaluate the relatively high-quality factor $Q=\frac{\Delta f}{\delta f}=\frac{\pi R_{K}}{16 \sqrt{3} K Z_{s}} \simeq \frac{60}{K}$.

\section{BEYOND THE BALLISTIC LIMIT}

The devices previously introduced provide direct simultaneous access to $v$ and $K$. One can, however, wonder how scattering in the channels could alter the previous results. In this framework of transmission lines, a basic model of scattering is proposed via an additional line resistance, $\mathcal{R}_{e}$, in series with $\mathcal{C}_{e}$. To lowest order [38], the propagation term $s_{ \pm}$ then acquires an additional exponential term, $e^{-\frac{\mathcal{R}_{e}}{2 Z_{e}} d}$. It is then straightforward to show that the linear resonator exhibits a modified saturation value, $\left|T_{\text {sat }}\right|=\frac{8 K Z_{g}}{R_{K}} e^{-\frac{\mathcal{R}_{e} d}{2 Z_{e}}}$, while the phase $\arg (T)$ is not modified. The change of $\left|T_{\text {sat }}\right|$ is illustrated in Fig. 5(a) for loss parameters recently measured in $\mathrm{HgTe}$ layers [49] for which $\mathcal{R}_{e}$ ranges from $\sim 0.1$ to $\sim 3 \mathrm{k} \Omega \mu \mathrm{m}^{-1}$. In the ring resonator, the FP peaks are more strongly modified. They have a decreased maximum transmission $\left|T_{\max }\right|<1$ and an increased width $\delta f$ that read as follows:

$$
\begin{gathered}
\left|T_{\max }\right|=\frac{8 Z_{g} e^{-\frac{\pi \mathcal{R}_{e r}}{Z_{e}}}}{Z_{e}\left(1-e^{-\frac{\pi \mathcal{R}_{e} r}{\mathcal{Z}_{e}}}\right)+Z_{g}\left(1+e^{-\frac{\pi \mathcal{R}_{e} r}{\mathcal{Z}_{e}}}\right)}, \\
\delta f=\frac{v \sqrt{3}}{2 \pi^{2} r}\left[\left(1+\frac{16 K Z_{g}}{R_{K}}\right) e^{\frac{\pi \mathcal{R}_{e r} r}{Z_{e}}}-1\right] .
\end{gathered}
$$


(a)

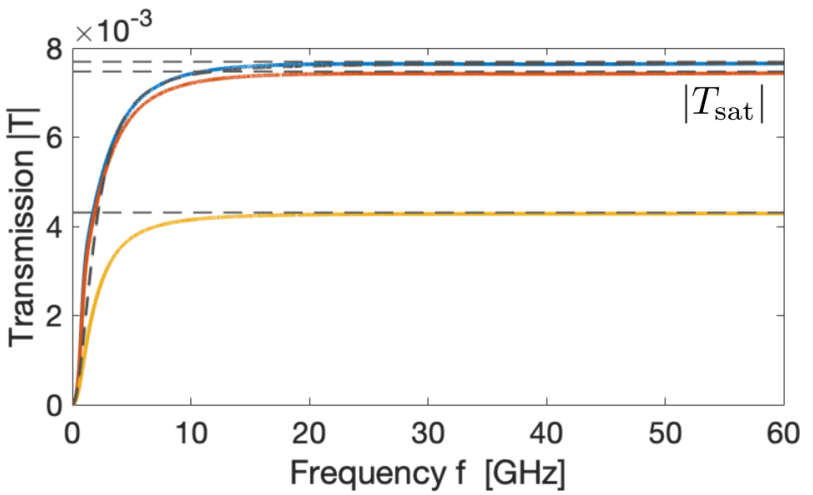

(b)

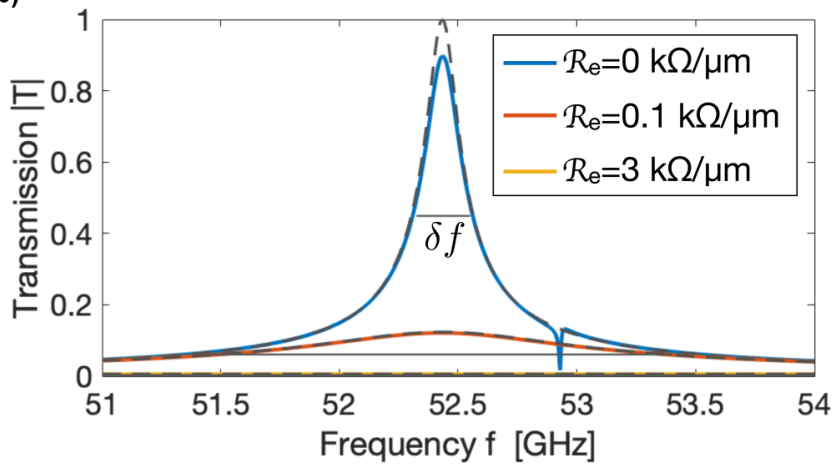

FIG. 5. Effect of losses on the transmission. (a) For $\mathcal{R}_{e}=0,0.1$, and $3 \mathrm{k} \Omega \mu \mathrm{m}^{-1}$, the moduli $|T|$ of the transmission of the linear resonator are plotted as solid colored lines. The dashed lines represent the high-frequency asymptote $\left|T_{\text {sat }}\right|$. (b) For $\mathcal{R}_{e}=0,0.1$, and $3 \mathrm{k} \Omega$ $\mu \mathrm{m}^{-1}$, the moduli $|T|$ of the transmission of the ring resonators are plotted as solid colored lines. The dashed lines represent the approximation given in Eq. (17), while the solid gray lines represent the peak widths $\delta f$. All graphs were obtained with the following parameters: $C_{e}=16 \mathrm{fF}, C_{g}=8 \mathrm{fF}, v=1 \times 10^{6} \mathrm{~ms}^{-1}, K=0.5, d=10 \mu \mathrm{m}$, and $r=6 \mu \mathrm{m}$.

As a consequence, in Fig. 5(b), we observe that a finite $\mathcal{R}_{e}$ strongly suppresses the Fabry-Pérot resonances in the ring resonator. From Eq. (17), one notices that, for large $\mathcal{R}_{e}$, the featureless transmission $T$ is then analogous to that of the linear resonator, with $|T| \rightarrow \frac{16 K Z_{g}}{R_{K}} e^{-\frac{2 \pi r \mathcal{Z}_{e}}{2 T_{e}}}$. In any case, measurements of samples with different travel lengths should be employed to carefully take this effect into account.

\section{EXPERIMENTAL CONSIDERATIONS}

Finally, we review the conditions required to perform the experiments. First, we note the impedance mismatch which allows for determining $K$ yields rather small values of $\left|T_{\text {sat }}\right| \simeq$ $0.005 \simeq-45 \mathrm{~dB}$. Though small, these values are customary when measuring edge states in the microwave regime [41] and remain experimentally measurable with standard vector network analyzers (VNAs).

Second, we stress that we have restricted this study to short distances (here $d=10 \mu \mathrm{m}, r=6 \mu \mathrm{m}$ ) so that the edge states remain as close as possible to the ballistic limit, usually 5 to $10 \mu \mathrm{m}$ [49-51]. Besides, in a recent study of HgTe quantum wells [41], we have measured the quantum capacitance, which remains large even in the topological gap. From the data, we estimate that $v \gtrsim v_{F} \simeq 1 \times 10^{6} \mathrm{~ms}^{-1}, C_{g} \simeq 8 \mathrm{fF}$, and $C_{e}=16 \mathrm{fF}$ for a gate with an area of $\mathcal{A}=2 \mu \mathrm{m}^{2}$. Given these parameters, the high-frequency range of the study identified in Figs. 2 and 4 lies beyond $20 \mathrm{GHz}$. This is challenging but not inaccessible thanks to the development of cryogenic microwave probe stations. They allow measurements down to $4 \mathrm{~K}$ and up to $67 \mathrm{GHz}[52,53]$, with accurate in situ microwave calibration. Besides, these parameters ensure that the pointlike description of the gate coupling is sufficient, as the finite length of the gate $\sqrt{\mathcal{A}}$ is always much smaller than the plasmon wavelength in the whole frequency range, i.e., such that $\sqrt{\mathcal{A}} \ll v / f$.

In other systems, with smaller velocities [54], or when quantum and geometric capacitances are smaller, larger gates may be necessary. In that case, the transmission line approach with pointlike contacts between gate and edge states in principle fails. A long-range distributed gate coupling can be worked out based on Ref. [55]. The results described here can then be adapted to take into account the additional effects (propagation and finite-size effects) arising [56], and the validity of our approach is not questioned.

Finally, we point out that the presence of a metallic topgate (which screens interactions) and its distance to the helical edge channels may significantly modify the value of $v$ and $K$. Several configurations (back gate, top gate, and resistive top gate [57]) could thus be tested. In combination with dc measurements, one could also assess the influence of Coulomb interactions on scattering in helical edge channels, as suggested by many theoretical works [8-10].

\section{CONCLUSION}

As a conclusion, we have used the analogy between helical Luttinger liquids of a QSH insulator and microwave transmission lines to develop simple models of helical Luttinger liquids and their coupling to local capacitive contacts. We have combined these building blocks in two different resonator geometries and have shown that the measurement of the microwave transmission coefficient $T$ allows for a very natural determination of the velocity $v$ and the Luttinger parameter $K$. Thus, a full characterization of Coulomb interaction can be obtained, shining new light on its consequences on the dynamics and back-scattering in helical edge states. The challenge resides in the use of high frequencies $(>10 \mathrm{GHz})$, now readily accessible in cryogenic microwave probe stations, in combination with conventional VNAs.

We believe that the general framework developed here for helical liquids can be extended to other types of interacting 1D systems, such as the chiral edge states of the integer and fractional quantum Hall effects (though the geometries studied in this article are irrelevant). Future developments will thus aim at proposing geometries that will unveil the exact nature of modes (charged vs neutral modes, Majorana edge states, etc.) and allow better understanding of the effects of edge reconstructions.

The data sets generated and analyzed during the current study are available from the corresponding author upon reasonable request. 


\section{ACKNOWLEDGMENTS}

This work has been supported by the European Research Council (ERC), under Contract No. ERC-2017-StG 758077 "CASTLES." E.B. gratefully acknowledges enlightening dis- cussions with R. Abdellatif. A.G., H.K., and E.B. proposed the model and performed the analytical and numerical analysis. E.B. supervised the project. All authors participated in the analysis of the results and in the writing of the manuscript.
[1] M. Konig, S. Wiedmann, C. Brune, A. Roth, H. Buhmann, L. W. Molenkamp, X.-L. Qi, and S.-C. Zhang, Quantum spin Hall insulator state in HgTe quantum wells, Science 318, 766 (2007).

[2] A. Roth, C. Brune, H. Buhmann, L. W. Molenkamp, J. Maciejko, X.-L. Qi, and S.-C. Zhang, Nonlocal transport in the quantum spin Hall state, Science 325, 294 (2009).

[3] I. Knez, R.-R. Du, and G. Sullivan, Evidence for Helical Edge Modes in Inverted InAs/GaSb Quantum Wells, Phys. Rev. Lett. 107, 136603 (2011).

[4] C. Brüne, A. Roth, H. Buhmann, E. M. Hankiewicz, L. W. Molenkamp, J. Maciejko, X.-L. Qi, and S.-C. Zhang, Spin polarization of the quantum spin Hall edge states, Nat. Phys. 8, 485 (2012).

[5] E. Bocquillon, R. S. Deacon, J. Wiedenmann, P. Leubner, T. M. Klapwijk, C. Brüne, K. Ishibashi, H. Buhmann, and L. W. Molenkamp, Gapless Andreev bound states in the quantum spin Hall insulator HgTe, Nat. Nanotechnol. 12, 137 (2017).

[6] R. S. Deacon, J. Wiedenmann, E. Bocquillon, F. Domínguez, T. M. Klapwijk, P. Leubner, C. Brüne, E. M. Hankiewicz, S. Tarucha, K. Ishibashi, H. Buhmann, and L. W. Molenkamp, Josephson Radiation from Gapless Andreev Bound States in HgTe-Based Topological Junctions, Phys. Rev. X 7, 021011 (2017).

[7] C. Wu, B. A. Bernevig, and S.-C. Zhang, Helical Liquid and the Edge of Quantum Spin Hall Systems, Phys. Rev. Lett. 96, 106401 (2006).

[8] J. Maciejko, C. Liu, Y. Oreg, X.-L. Qi, C. Wu, and S.-C. Zhang, Kondo Effect in the Helical Edge Liquid of the Quantum Spin Hall State, Phys. Rev. Lett. 102, 256803 (2009).

[9] F. Crépin, J. C. Budich, F. Dolcini, P. Recher, and B. Trauzettel, Renormalization group approach for the scattering off a single Rashba impurity in a helical liquid, Phys. Rev. B 86, 121106(R) (2012).

[10] C.-H. Hsu, P. Stano, J. Klinovaja, and D. Loss, Nuclearspin-induced localization of edge states in two-dimensional topological insulators, Phys. Rev. B 96, 081405(R) (2017).

[11] D. Pesin and A. H. MacDonald, Spintronics and pseudospintronics in graphene and topological insulators, Nat. Mater. 11, 409 (2012)

[12] P. P. Hofer, H. Aramberri, C. Schenke, and P. A. L. Delplace, Proposal for an ac spin current source, Europhys. Lett. 107, 27003 (2014).

[13] I. Safi and H. J. Schulz, Transport in an inhomogeneous interacting one-dimensional system, Phys. Rev. B 52, R17040(R) (1995).

[14] D. L. Maslov and M. Stone, Landauer conductance of Luttinger liquids with leads, Phys. Rev. B 52, R5539(R) (1995).

[15] R. Stühler, F. Reis, T. Müller, T. Helbig, T. Schwemmer, R. Thomale, J. Schäfer, and R. Claessen, Tomonaga-Luttinger liquid in the edge channels of a quantum spin Hall insulator, Nat. Phys. 16, 47 (2020).
[16] T. Li, P. Wang, H. Fu, L. Du, K. A. Schreiber, X. Mu, X. Liu, G. Sullivan, G. A. Csáthy, X. Lin, and R.-R. Du, Observation of a Helical Luttinger Liquid in InAs/GaSb Quantum Spin Hall Edges, Phys. Rev. Lett. 115, 136804 (2015).

[17] Y. Sato, S. Matsuo, C.-H. Hsu, P. Stano, K. Ueda, Y. Takeshige, H. Kamata, J. S. Lee, B. Shojaei, K. Wickramasinghe, J. Shabani, C. Palmstrøm, Y. Tokura, D. Loss, and S. Tarucha, Strong electron-electron interactions of a Tomonaga-Luttinger liquid observed in InAs quantum wires, Phys. Rev. B 99, 155304 (2019).

[18] B. Trauzettel, I. Safi, F. Dolcini, and H. Grabert, Appearance of Fractional Charge in the Noise of Nonchiral Luttinger Liquids, Phys. Rev. Lett. 92, 226405 (2004).

[19] I. Safi, C. Bena, and A. Crépieux, ac conductance and nonsymmetrized noise at finite frequency in quantum wires and carbon nanotubes, Phys. Rev. B 78, 205422 (2008).

[20] Y. M. Blanter, F. W. J. Hekking, and M. Buttiker, Interaction Constants and Dynamic Conductance of a Gated Wire, Phys. Rev. Lett. 81, 1925 (1998).

[21] T. Müller, R. Thomale, B. Trauzettel, E. Bocquillon, and O. Kashuba, Dynamical transport measurement of the Luttinger parameter in helical edges states of 2D topological insulators, Phys. Rev. B 95, 245114 (2017).

[22] H. Kamata, N. Kumada, M. Hashisaka, K. Muraki, and T. Fujisawa, Fractionalized wave packets from an artificial Tomonaga-Luttinger liquid, Nat. Nanotechnol. 9, 177 (2014).

[23] P. Brasseur, N. H. Tu, Y. Sekine, K. Muraki, M. Hashisaka, T. Fujisawa, and N. Kumada, Charge fractionalization in artificial Tomonaga-Luttinger liquids with controlled interaction strength, Phys. Rev. B 96, 081101(R) (2017).

[24] R. C. Ashoori, H. L. Stormer, L. N. Pfeiffer, K. W. Baldwin, and K. West, Edge magnetoplasmons in the time domain, Phys. Rev. B 45, 3894 (1992).

[25] N. B. Zhitenev, R. J. Haug, K. v. Klitzing, and K. Eberl, TimeResolved Measurements of Transport in Edge Channels, Phys. Rev. Lett. 71, 2292 (1993).

[26] G. Sukhodub, F. Hohls, and R. J. Haug, Observation of an Interedge Magnetoplasmon Mode in a Degenerate TwoDimensional Electron Gas, Phys. Rev. Lett. 93, 196801 (2004).

[27] J. Gabelli, G. Fève, T. Kontos, J.-M. Berroir, B. Plaçais, D. C. Glattli, B. Etienne, Y. Jin, and M. Büttiker, The Relaxation Time of a Chiral Quantum $R-L$ Circuit, Phys. Rev. Lett. 98, 166806 (2007).

[28] H. Kamata, T. Ota, K. Muraki, and T. Fujisawa, Voltagecontrolled group velocity of edge magnetoplasmon in the quantum Hall regime, Phys. Rev. B 81, 085329 (2010).

[29] M. Hashisaka, H. Kamata, N. Kumada, K. Washio, R. Murata, K. Muraki, and T. Fujisawa, Distributed-element circuit model of edge magnetoplasmon transport, Phys. Rev. B 88, 235409 (2013). 
[30] N. Kumada, H. Kamata, and T. Fujisawa, Edge magnetoplasmon transport in gated and ungated quantum hall systems, Phys. Rev. B 84, 045314 (2011).

[31] E. Bocquillon, V. Freulon, J.-M. Berroir, P. Degiovanni, B. Plaçais, A. Cavanna, Y. Jin, and G. Fève, Separation of neutral and charge modes in one-dimensional chiral edge channels, Nat. Commun. 4, 1839 (2013).

[32] N. Kumada, P. Roulleau, B. Roche, M. Hashisaka, H. Hibino, I. Petković, and D. C. Glattli, Resonant Edge Magnetoplasmons and Their Decay in Graphene, Phys. Rev. Lett. 113, 266601 (2014).

[33] V. Freulon, A. Marguerite, J.-M. Berroir, B. Plaçais, A. Cavanna, Y. Jin, and G. Fève, Hong-Ou-Mandel experiment for temporal investigation of single-electron fractionalization, Nat. Commun. 6, 6854 (2015).

[34] A. C. Mahoney, J. I. Colless, S. J. Pauka, J. M. Hornibrook, J. D. Watson, G. C. Gardner, M. J. Manfra, A. C. Doherty, and D. J. Reilly, On-Chip Microwave Quantum Hall Circulator, Phys. Rev. X 7, 011007 (2017).

[35] A. C. Mahoney, J. I. Colless, L. Peeters, S. J. Pauka, E. J. Fox, X. Kou, L. Pan, K. L. Wang, D. Goldhaber-Gordon, and D. J. Reilly, Zero-field edge plasmons in a magnetic topological insulator, Nat. Commun. 8, 1836 (2017).

[36] G. Viola and D. P. DiVincenzo, Hall Effect Gyrators and Circulators, Phys. Rev. X 4, 021019 (2014).

[37] Q. Wilmart, A. Inhofer, M. Boukhicha, W. Yang, M. Rosticher, P. Morfin, N. Garroum, G. Fève, J.-M. Berroir, and B. Plaçais, Contact gating at $\mathrm{GHz}$ frequency in graphene, Sci. Rep. 6, 21085 (2016)

[38] D. Pozar, Microwave Engineering (Wiley \& Sons, New York, 2012).

[39] E. Pallecchi, A. C. Betz, J. Chaste, G. Fève, B. Huard, T. Kontos, J.-M. Berroir, and B. Plaçais, Transport scattering time probed through rf admittance of a graphene capacitor, Phys. Rev. B 83, 125408 (2011).

[40] A. Inhofer, S. Tchoumakov, B. A. Assaf, G. Fève, J. M. Berroir, V. Jouffrey, D. Carpentier, M. O. Goerbig, B. Plaçais, K. Bendias, D. M. Mahler, E. Bocquillon, R. Schlereth, C. Brüne, H. Buhmann, and L. W. Molenkamp, Observation of Volkov-Pankratov states in topological HgTe heterojunctions using high-frequency compressibility, Phys. Rev. B 96, 195104 (2017).

[41] M. C. Dartiailh, S. Hartinger, A. Gourmelon, K. Bendias, H. Bartolomei, H. Kamata, J.-M. Berroir, G. Fève, B. Plaçais, L. Lunczer, R. Schlereth, H. Buhmann, L. W. Molenkamp, and E. Bocquillon, Dynamical Separation of Bulk and Edge Transport in HgTe-Based 2D Topological Insulators, Phys. Rev. Lett. 124, 076802 (2020).

[42] R. Tarkiainen, M. Ahlskog, J. Penttilä, L. Roschier, P. Hakonen, M. Paalanen, and E. Sonin, Multiwalled carbon nanotube: Luttinger versus Fermi liquid, Phys. Rev. B 64, 195412 (2001).
[43] P. Burke, Luttinger liquid theory as a model of the gigahertz electrical properties of carbon nanotubes, IEEE Trans. Nanotechnol. 1, 129 (2002).

[44] G. Dolcetto, M. Sassetti, and T. L. Schmidt, Edge physics in two-dimensional topological insulators, La Rivista del Nuovo Cimento 39, 113 (2016).

[45] We note that this approach also includes gate screening effects, simply by renormalizing $\mathcal{C}_{e}$.

[46] G. Feve, A. Mahe, J.-M. Berroir, T. Kontos, B. Placais, D. C. Glattli, A. Cavanna, B. Etienne, and Y. Jin, An ondemand coherent single-electron source, Science 316, 1169 (2007).

[47] S. Bosco and D. P. DiVincenzo, Non-reciprocal quantum Hall devices with driven edge magnetoplasmons in two-dimensional materials, Phys. Rev. B 95, 195317 (2017).

[48] To avoid confusions, we recall that, unlike here, scattering matrices are usually defined for modes or voltages [38] .

[49] L. Lunczer, P. Leubner, M. Endres, V. L. Müller, C. Brüne, H. Buhmann, and L. W. Molenkamp, Approaching Quantization in Macroscopic Quantum Spin Hall Devices through Gate Training, Phys. Rev. Lett. 123, 047701 (2019).

[50] L. Du, I. Knez, G. Sullivan, and R.-R. Du, Robust Helical Edge Transport in Gated InAs/GaSb Bilayers, Phys. Rev. Lett. 114, 096802 (2015).

[51] K. Bendias, S. Shamim, O. Herrmann, A. Budewitz, P. Shekhar, P. Leubner, J. Kleinlein, E. Bocquillon, H. Buhmann, and L. W. Molenkamp, High mobility HgTe microstructures for quantum spin Hall studies, Nano Lett. 18, 4831 (2018).

[52] H. Graef, D. Mele, M. Rosticher, L. Banszerus, C. Stampfer, T. Taniguchi, K. Watanabe, E. Bocquillon, G. Fève, J.-M. Berroir, E. H. T. Teo, and B. Plaçais, Ultra-long wavelength Dirac plasmons in graphene capacitors, J. Phys.: Mater. 1, 01LT02 (2018).

[53] H. Graef, Dirac fermion optics and plasmonics in graphene microwave devices, Ph.D. thesis, Sorbonne Université-Nanyang Technological University, 2019.

[54] L. Du, T. Li, W. Lou, X. Wu, X. Liu, Z. Han, C. Zhang, G. Sullivan, A. Ikhlassi, K. Chang, and R.-R. Du, Tuning Edge States in Strained-Layer InAs/GaInSb Quantum Spin Hall Insulators, Phys. Rev. Lett. 119, 056803 (2017).

[55] P. Degiovanni, C. Grenier, and G. Fève, Decoherence and relaxation of single electron excitations in quantum Hall edge channels, Phys. Rev. B 80, 241307(R) (2009).

[56] T. Ota, M. Hashisaka, K. Muraki, and T. Fujisawa, Electronic energy spectroscopy of monochromatic edge magnetoplasmons in the quantum Hall regime, J. Phys.: Condens. Matter 30, 345301 (2018).

[57] N. Kumada, N.-H. Tu, K.-i. Sasaki, T. Ota, M. Hashisaka, S. Sasaki, K. Onomitsu, and K. Muraki, Suppression of gate screening on edge magnetoplasmons by highly resistive $\mathrm{ZnO}$ gate, Phys. Rev. B 101, 205205 (2020). 